\title{
The Effect of Peer Education on Fear of Childbirth in Pregnant Women: A Randomized Clinical Trial
}

\author{
Roghayeh Rahmani ${ }^{1}$, Fatemeh Yaghoobi Moghadam ${ }^{1 *}$, Fatemeh Hadizadeh-Talasaz ${ }^{1}$, Mohamad Reza \\ Rahmani $^{2}$
}

\begin{abstract}
Objectives: The fear of childbirth (FOC) is a common problem in women's lives. Several interventions were carried out to reduce the fear of delivery during pregnancy. Despite these interventions, the increase in cesarean statistics indicates that routine interventions are not effective in controlling the FOC. Accordingly, the present study investigated the effect of peer education on the degree of FOC in pregnant women.

Materials and Methods: This clinical trial was performed on 108 low-risk pregnant women aged 18-35 years old referring to community health centers of Gonabad University of Medical Sciences. The participants were randomly assigned to peer education, discussion, and control groups using block randomization (36 in each group). The peer education and discussion groups included four 2-hour sessions for 4 weeks and a training booklet. In peer education and discussion groups, the training was done by trained counterparts and a specialist, respectively. Finally, the data were analyzed using SPSS software through ANOVA and chi-square tests. Results: The results showed a significant difference between mean scores of the FOC in the 3 groups after the intervention $(P=0.007)$. In addition, the Scheffe test showed a significant difference between peer education and the discussion groups $(P=0.013)$. However, after 4 weeks of intervention, no significant difference was observed in the choice of delivery type in the 3 groups $(P=0.122)$.

Conclusions: In general, the interventional therapy program of peer education is effective in decreasing the FOC in pregnant women. Keywords: Peer group, Education, Fear, Childbirth
\end{abstract}

\section{Introduction}

Fear of childbirth (FOC) is a well-known problem in women's lives that affects the outcome of pregnancy (1). It is estimated that one out of 5 pregnant women has FOC and about $6 \%-13 \%$ of pregnant women are seized by a severe and disabling fear which is associated with labor (2). The factors affecting the FOC include women's personal, internal and external conditions (i.e., poor mental health, anxiety disorders, and previous experiences of trauma and abuse), as well as social circumstances such as insufficient social support, unemployment and economic problems which are of importance in FOC (3). In addition, FOC makes the physiological flow childbirth abnormal (4). Mothers who are afraid of having a childbirth have problems with mother-child compliance $(5,6)$. Other possible complications of fears include abnormal fetal heart rate, low Apgar score in the infant, increased mortality during birth, low birth weight due to maternal anxiety, increased resistance of the uterine arteries in addition to the increased pain in the mother due to hypoxia because of decreased blood flow to the pelvic muscles in response to increased catecholamines and serum cortisol (7).

Further, FOC can lead to posttraumatic stress disorders that are considered as responses to negative birth experiences and influence women not only following pregnancies but also during and after birth. Posttraumatic stress disorders can affect the relationship between the mother and the baby in postpartum stages as well (8).

The fear of pain and feeling of the mother's inability to tolerate the delivery process are the reasons for increasing the cesarean section, which tends to reduce maternal delivery pain by accepting delivery via cesarean section. Different methods, including psychological counseling, musical use, cognitive-behavioral therapy, and communication with people who have previously been under the same conditions (9), are used to reduce the FOC. In recent years, more emphasis has been placed on the positive and constructive role of a person in the creation, maintenance, and promotion of health, as well as the fact that a significant part of health behaviors is the result of learning from others, and in particular, the effects of peer groups. Thus, the use of a peer education approach, which is considered a behavior change strategy, can be applied in this regard (10). In a peer education approach, the peers communicate with other peers and transfer the information effectively thus affecting their 
counterparts (11).

In the peer education process, individuals' empowerment for taking over responsibility and protecting their health is considered a target in addition to developing their level of knowledge, attitude, beliefs, and skills (10-12). In peer education, the peer and patient's membership in a group strengthens the sense of empathy and social identity and leads to increased knowledge (13). In this case, patients find their peers more comfortable and share their secrets with them (14). Considering the high prevalence of childbirth fear in pregnant women in Iran and an increase in the choice of unnecessary cesarean delivery, the need for new, easy, and low-cost educational interventions, including the effect of education through peers on the FOC, seems necessary.

\section{Materials and Methods \\ Participants}

The sample included 108 pregnant women who were randomly assigned to 3 groups. The inclusion criteria were being in the age range of 18-35 years old and the gestational age of 24-34 weeks, having a low-risk pregnancy, obtaining a score of less than 85 for FOC before entering the study, not having the history of dystocia, postpartum bleeding and fetal abnormalities, showing the willingness for participation in the study, having reading and writing skills in Persian, lacking any physical and mental illness, and not having indicators for cesarean section in accordance with the Mothers' Electronic-Health Record.

The exclusion criteria were the absence or irregular presence in the sessions, the incidence of any complications of pregnancy (e.g., preeclampsia, bleeding, diabetes, intrauterine death, and preterm delivery), history of physical injury caused by accidents, beatings, violence, along with falling from a height in person or in his family. Moreover, mothers who were not willing to continue with the study at any stage were free to leave the study.

\section{Design}

This randomized controlled clinical trial study included all pregnant women with an electronic health record in community health centers affiliated to Gonabad University of Medical Sciences during 2017-2018.

\section{Sample Size}

According to Ghazaie et al (15), the sample size was calculated as 36 in each group considering a drop probability of $15 \%(\alpha=0.05$ and $\beta=0.1)$. The samples were randomly assigned to 3 groups using a blocking method.

\section{Data Collection Tool}

The data collection tools included:

1. A demographic questionnaire: The first part contained personal, social, and general information and the second part included selecting the type of delivery and pregnancy information.

2. Widget's Maternity Fear Awareness Questionnaire: This questionnaire contained 33 questions based on a 5-point Likert-type scale ranging from "completely" to "at all" (0-5). A minimum score of zero and a maximum score of 165 were calculated in which the scores less than or equal to $37,38-65$, and $84-66$ indicated mild, average, and severe fear, respectively. In addition, the cutting score was 85 , namely, the scores above 85 proved clinical scrutiny. Mortazavi in Iran estimated the Cronbach $\alpha$ as 0.91 (16).

\section{Intervention}

The pregnant mothers willing to participate in the study completed a checklist of inclusion criteria, and they were selected accordingly. The mothers who possessed the inclusion criteria were invited, the research objectives were briefed to them, followed by obtaining the informed consent forms. The 2-stage sampling was used in this study. First, a convenient sampling method was performed to determine the research population. Then, they were randomly assigned to 3 groups using a quadruple blocking method. Research blindness was done by placing pregnant mothers in peer education, discussion, and control groups based on the list of blocking with the researcher's assistance. A flow chart of the study is shown in Figure 1.

Based on the method of plan execution, the pregnant mothers were divided into 3 groups (2 intervention groups and one control group). The intervention groups in this study included:

1. Peer teaching group: The training lasted for 4 twohour sessions during 4 days for a period of 4 weeks and in the peer group meeting with the attendance of an eligible trainee counterpart;

2. Group discussion group: The training sessions were conducted with the presence of a specialist for 4 twohour sessions for 4 days for a period of 4 weeks.

The content of the training included the nature of delivery, labor pain, and the methods for reducing FOC (Table 1). The sample selection criteria for the peer educator encompassed having a history of at least one physiological delivery during the past year, passing the training courses, and gaining a passing grade. It should be noted that all peers completed training courses under the supervision of an expert. To select the trained counterpart, the following procedure was carried out.

1. Calling for co-sponsoring qualified mothers;

2. Registering 20 eligible mothers;

3. Holding training courses (Table 2);

4. Giving the test;

5. Selecting eight mothers as educator counterparts.

\section{Results}

Five pregnant women were excluded from the study, therefore, 103 pregnant women were evaluated in the current study, most of whom were within the age range of 


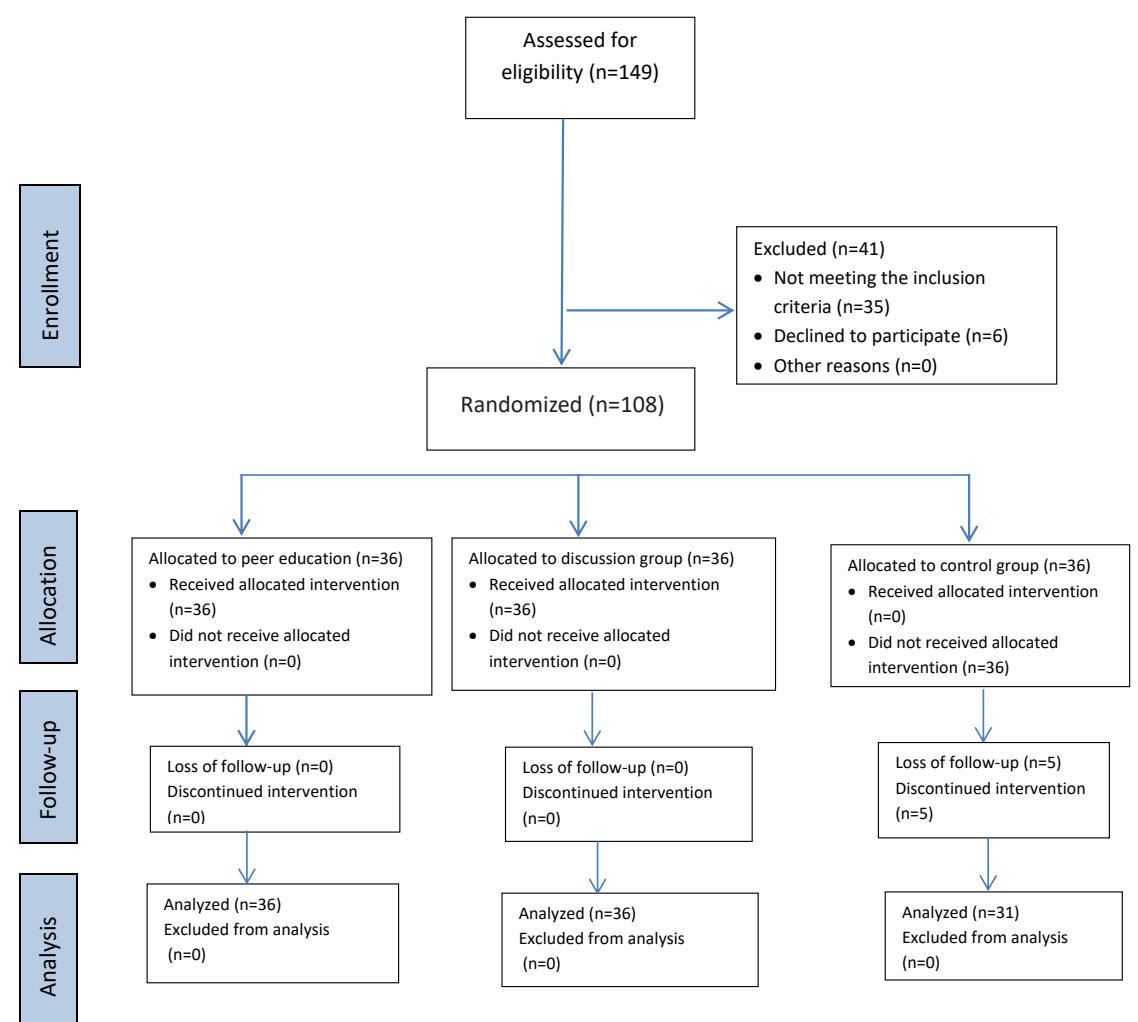

Figure 1. The Flow Diagram of Study (17).

19-28 years old. The maximum education was a diploma, the data normalization was confirmed by using the Shapiro statistical test, and finally, demographic and pregnancy data were matched in 3 groups (Table 3 ). At the beginning, there was no significant difference between the scores of fear in the 3 groups $(P=0.551)$, but the fear scores were compared in 3 groups and the obtained results displayed a significant difference among the 3 groups $(P=0.007)$ after
4 weeks of intervention (Table 4).

Further, the Scheffe test showed a significant difference between the peer education and control groups $(P=0.04)$, as well as the training and discussion groups with the peer education group $(P=0.013)$. However, no significant difference was observed in choosing the type of delivery in the 3 groups before intervention $(P=0.620)$ and after the intervention $(P=0.122)$.

Table 1. Training Session Group Discussion and Peer Education

\begin{tabular}{ll}
\hline Session & Educational Contents \\
\hline 1 & Getting familiar with the other peers, aims, and objectives, the general FOC, and overview; \\
2 & The care pregnancy, the mechanism and stages of delivery with questions and answers and the presentation of the film of childbirth; \\
3 & $\begin{array}{l}\text { The mechanism of labor pain and its reduction methods and the advantages of normal delivery compared with the cesarean section } \\
\text { with questions and answers; }\end{array}$ \\
\hline
\end{tabular}

Table 2. Training Session Trained Counterpart

\begin{tabular}{ll}
\hline Session & Educational Contents \\
\hline 1 & $\begin{array}{l}\text { Welcoming, emphasizing the importance of peer education and communication skills such as non-verbal behaviors, the ability to } \\
\text { receive and send clear communication messages, and active listening; }\end{array}$ \\
& $\begin{array}{l}\text { The mechanism and stages of delivery, obstetric care and pregnancy, questions and answers, the use of supplements in pregnancy, } \\
\text { and nutrition in pregnancy; }\end{array}$ \\
3 & $\begin{array}{l}\text { The mechanism of labor pain and its reduction methods, the advantages of normal delivery compared with the cesarean section with } \\
4\end{array}$ \\
\end{tabular}


Table 3. Demographic Characteristics of Participants of the Study

\begin{tabular}{|c|c|c|c|c|}
\hline & Peer Education & Discussion Group & Control Group & P Value $^{c}$ \\
\hline \multicolumn{4}{|l|}{ Age $(y)$} & \multirow{2}{*}{0.121} \\
\hline $18-35$ & $24.4(4.14)$ & $26.52^{\mathrm{a}}(4.6)^{\mathrm{b}}$ & $25.6(4.35)$ & \\
\hline \multicolumn{5}{|l|}{ Education } \\
\hline High school & $14(38.9)$ & $14(38.9)$ & $11(45.5)$ & \multirow{3}{*}{0.08} \\
\hline Diploma & $18(50)$ & $14(38.9)$ & $13(41.9)$ & \\
\hline Academic & 4 (11.1) & $8(22.2)$ & $7(22.6)$ & \\
\hline \multicolumn{5}{|l|}{ Job } \\
\hline Housewife & $34(94.4)^{d}$ & $31(86.1)$ & $26(83.87)$ & \multirow{3}{*}{0.51} \\
\hline Employee & 0 & $5(13.88)$ & $5(16.12)$ & \\
\hline Others & $2(5.55)$ & 0 & 0 & \\
\hline \multicolumn{5}{|l|}{ Location } \\
\hline City & $29(88.55)$ & $26(72.22)$ & $22(70.96)$ & \multirow{2}{*}{0.667} \\
\hline Village & $7(19.45)$ & $10(27.78)$ & $9(29.04)$ & \\
\hline \multicolumn{5}{|l|}{ Gravida } \\
\hline Primi-gravid & $16(44.44)$ & $20(55.55)$ & $16(51.61)$ & \multirow{2}{*}{0.77} \\
\hline Multi-gravid & $20(55.56)$ & $16(44.45)$ & 15 (48.39) & \\
\hline \multicolumn{5}{|l|}{ Abortion } \\
\hline No & $30(83.34)$ & 33 (91.67) & $28(90.33)$ & \multirow{2}{*}{0.5} \\
\hline Yes & $6(16.67)$ & $3(8.33)$ & $3(9.68)$ & \\
\hline \multicolumn{5}{|l|}{ Infertility } \\
\hline Yes & $1(2.77)$ & $3(8.34)$ & $3(9.67)$ & \multirow{2}{*}{0.78} \\
\hline No & $35(97.23)$ & 33 (91.67) & $28(90.33)$ & \\
\hline \multicolumn{5}{|c|}{ Pregnancy condition } \\
\hline Wanted & $29(80.55)$ & $32(88.88)$ & $27(87 / 09)$ & \multirow{2}{*}{0.72} \\
\hline Unwanted & $7(19.45)$ & $4(11.12)$ & $4(12.91)$ & \\
\hline
\end{tabular}

Note. ${ }^{a}$ Mean; ${ }^{b}$ Standard deviation (SD); ${ }^{\mathrm{c}} P<0.05$ was considered significant; ${ }^{\mathrm{d}}$ Values are expressed as frequency (\%).

Table 4. Comparison of Mean Fear Scores of Pregnant Women in Peer Education, Discussion, and Control Groups ${ }^{\mathrm{a}}$

\begin{tabular}{lccc}
\hline Severity of Fear & Peer Education Group & Discussion Group & Control Group \\
\hline Before education & $81.95(13.7)$ & $78.8(20.9)$ & $77.1(20.72)$ \\
Four weeks after education & $68.2(22.5)$ & $69.9(15.1)$ & $80.23(10.63)$ \\
\hline
\end{tabular}

Note. ${ }^{\text {a } V a l u e s ~ a r e ~ e x p r e s s e d ~ a s ~ m e a n ~(S D) . ~}$

\section{Discussion}

The results of this study showed that peer education has an effect on reducing the FOC in pregnant women. Moreover, the peer education program was more effective in comparison with the discussion group. The mean scores of mothers' fear in the peer education group after the intervention decreased compared to their mean scores before the intervention. Furthermore, the fear of delivery in the discussion group reduced after the implementation of the educational program. However, the scores of the FOC in the peer education group decreased more than those of the discussion group. Women's fear of their health under unknown circumstances is the result of lack of awareness and education can reduce it (18).

In a study conducted by Mortazavi et al, childbirth fear and its associated factors were investigated in a sample of pregnant Iranian women. The results indicated a relatively high prevalence of childbirth fear in pregnant women in Iran (19).
The results of the study by Godarzi et al (20) demonstrated that lactation training through peer support increased lactation self-efficacy in nulliparous women.

Molazem et al (21) also found that using peer education is effective for reducing fear and anxiety in patients undergoing coronary angiography.

In another study, peer education was compared with nursing education on patients and the results showed that patients receiving peer education had higher self-efficacy compared to those who received nursing education (22).

In sum, most studies in the field of peer group indicate the positive and beneficial effects of this educational method are consistent with those of the present study. Moreover, the positive impact of this approach is probably due to the fact that the peer group transmits their experiences in a simple and effective manner and thus the pregnant women are more likely to be more confident in the experiences of those who have the same conditions as their own. 
In the present study, there was no significant correlation between the degree of fear among nulliparous women and multiparity. Nevertheless, the frequency of fear of childbirth was higher in multiparous women in a study conducted in Iran (23) while the results of a study abroad (24) demonstrated more fears in nulliparous women. The contradictions in these studies can be attributed to the difference in the female experience of childbirth, pregnancy training, and various social, as well as cultural and personality conditions.

Several studies have focused on group discussions. For instance, Tofighi Niaki et al (25) evaluated the effect of group training during pregnancy on knowledge and attitude of choosing the type of delivery in primiparous women. The results showed that there is a significant difference between women's knowledge and attitudes after training than before. Considering that people are in close interaction with each other and freely mention their positive or negative experiences in relation to their common theme in the group discussion method, those at the risk of injury can gain more social support. Additionally, they can make better decisions under certain conditions with the help of the experiences of women who are more or less like themselves. In the present study, the effect of counterparts on the fear of delivery had a more positive effect in comparison with education through group discussion. The greater impact of peer education can be due to the fact that the peers are a homogeneous group, all of whom almost share similar experiences about childbirth thus they understand each other better and state their problems more comfortably.

Based on the results of the present study, peer education group and discussion group had no positive effect on the choice of normal delivery by pregnant women. This can be attributed to the contribution of several factors such as social, cultural, psychosocial, and personality conditions, as well as the impact of family and community on the choice of delivery type, which are not controlled by scoring but require multi-dimensional interventions. The limitations of this research include the personality type of the individuals, their personality traits in the acceptance of teaching, and the accountability of individuals for their health, all of which should be taken into account in generalizing the results.

\section{Conclusions}

To the best of our knowledge, no studies have so far focused on the effect of peer education during pregnancy and childbirth. Therefore, more studies are suggested in this area. In addition, peer support models are a flexible, low-cost, and complementary supplement to health care, therefore, it is highly recommended to use such models in women's health policies and programs in the country.

\section{Conflict of Interests}

Authors declare that they have no conflict of interests.

\section{Ethical Issues}

The present study was approved by the Ethics Committee of Gonabad University of Medical Sciences under the number IR.KAUMS.REC.1397.007 and registered in the Iranian Registry of Clinical Trial (identifier: IRCT20180320039132N1; https://irct.ir/trial/30331). Written and informed consent was obtained from the participants as well.

\section{Financial Support}

This study was supported by the Social Development and Health Promotion Research Center, Gonabad University of Medical Sciences, Gonabad, Iran.

\section{Acknowledgments}

Hereby, the authors would like to thank the mothers who participated in the project, the peers who helped us, including Dr. Mostafa Amiri who edited the manuscript several times, as well as the Social Development and Health Promotion Research Center at Gonabad University of Medical Sciences, Gonabad, Iran.

\section{References}

1. Khwepeya M, Lee GT, Chen SR, Kuo SY. Childbirth fear and related factors among pregnant and postpartum women in Malawi. BMC Pregnancy Childbirth. 2018;18(1):391. doi:10.1186/s12884-018-2023-7

2. Alipour Z, Lamyian M, Hajizadeh E, Vafaei MA. The association between antenatal anxiety and fear of childbirth in nulliparous women: a prospective study. Iran J Nurs Midwifery Res. 2011;16(2):169-173.

3. Klabbers GA, van Bakel HJ, van den Heuvel M, Vingerhoets AJ. Severe fear of childbirth: Its features, assesment, prevalence, determinants, consequences and possible treatments. Psihol Teme. 2016;25(1):107-127.

4. Adams SS, Eberhard-Gran M, Eskild A. Fear of childbirth and duration of labour: a study of 2206 women with intended vaginal delivery. Bjog. 2012;119(10):1238-1246. doi:10.1111/j.1471-0528.2012.03433.x

5. Rouhe H, Salmela-Aro K, Toivanen R, et al. Group psychoeducation with relaxation for severe fear of childbirth improves maternal adjustment and childbirth experience--a randomised controlled trial. J Psychosom Obstet Gynaecol. 2015;36(1):1-9. doi:10.3109/0167482x.2014.980722

6. Salmela-Aro K, Read S, Rouhe H, et al. Promoting positive motherhood among nulliparous pregnant women with an intense fear of childbirth: RCT intervention. J Health Psychol. 2012;17(4):520-534. doi:10.1177/1359105311421050

7. Atghaee M, Nouhi E. The effect of imagination of the pain of vaginal delivery and cesarean section on the selection of normal vaginal delivery in pregnant women attending clinics in Kerman University of Medical Sciences. The Iranian Journal of Obstetrics, Gynecology and Infertility. 2012;14(7):44-50. doi:10.22038/ijogi.2012.6009

8. Ayers S, Bond R, Bertullies S, Wijma K. The aetiology of post-traumatic stress following childbirth: a meta-analysis and theoretical framework. Psychol Med. 2016;46(6):11211134. doi: $10.1017 / \mathrm{s} 0033291715002706$ 
9. Delaram M, Soltanpour F. The effect of counseling in third trimester on anxiety of nulliparous women at the time of admission for labor. Zahedan Journal of Research in Medical Sciences. 2012;14(2):61-65. [Persian].

10. United Nations Population Fund and Youth Peer Education Network. Training of Trainers Manual: From theory to practice in peer education. Arlington, USA: United Nations Population Fund; 2014.

11. Speizer IS, Magnani RJ, Colvin CE. The effectiveness of adolescent reproductive health interventions in developing countries: a review of the evidence. J Adolesc Health. 2003;33(5):324-348. doi:10.1016/s1054-139x(02)00535-9

12. Abedian Z, Kabirian M, Mazloum SR, Mahram B. Comparison of peer-led VS health provider-led self care education on severity of primary dysmenorrhea. Mashhad: Mashhad University of Medical Science; 2010.

13. Van Rompay KK, Madhivanan P, Rafiq M, Krupp K, Chakrapani V, Selvam D. Empowering the people: development of an HIV peer education model for low literacy rural communities in India. Hum Resour Health. 2008;6:6. doi:10.1186/1478-4491-6-6

14. Shah S, Peat JK, Mazurski EJ, et al. Effect of peer led programme for asthma education in adolescents: cluster randomised controlled trial. BMJ. 2001;322(7286):583-585. doi:10.1136/bmj.322.7286.583

15. Ghazaie M, Davoodi I, Neysi A, Mehrabizadeh Honarmand $\mathrm{M}$, Bassak Nejad S. The effectiveness of cognitive-behavioral therapy on fear of childbirth, fear of pain, self-efficacy of childbirth and tendency to caesarean in nulliparous women. The Iranian Journal of Obstetrics, Gynecology and Infertility. 2016;19(31):1-12. doi:10.22038/ijogi.2016.7989

16. Mortazavi F. Validity and reliability of the Farsi version of Wijma delivery expectancy questionnaire: an exploratory and confirmatory factor analysis. Electron Physician. 2017;9(6):4606-4615. doi:10.19082/4606

17. Schulz KF, Altman DG, Moher D. CONSORT 2010 statement: updated guidelines for reporting parallel group randomised trials. BMJ. 2010;340:c332. doi:10.1136/bmj. c332

18. Rahmani Bilandi R, Khalajabadi Farahamni F, Ahmadi F, Kazemnejad A, Mohammadi R. Exploring the Perception of Women about Menstrual Health in Earthquake Stricken Areas. J Mazandaran Univ Med Sci. 2015;25(125):49-60

19. Mortazavi F, Agah J. Childbirth fear and associated factors in a sample of pregnant Iranian women. Oman Med J. 2018;33(6):497-505. doi:10.5001/omj.2018.91

20. Godarzi Z, Saeidi M, Daneshvar Ameri Z, Shamshiri AR, Sadeghi T. Impact of peer education on breastfeeding self-efficacy in primiparous women: a cluster randomized controlled trial. Hakim Research Journal. 2015;18(2):105113. [Persian].

21. Molazem Z, Shahabfard Z, Askari A, Kalyani MN. Effects of a peer- led group education on fear, anxiety and depression levels of patients undergoing coronary angiography. Invest Educ Enferm. 2018;36(1):e13. doi:10.17533/udea.iee. v36n1e13

22. MohammadpourhodkiR, Rahnama M, Abdollahimohammad A, Shashraki Vahed A, Shamsizadeh M, Shahdadi H. Comparison of effect of nursing education and peer education methods on self-efficacy in patients with myocardial infarction. Mod Care J. 2018;15(1):e56012. doi:10.5812/ modernc. 56012

23. Dencker A, Nilsson C, Begley C, et al. Causes and outcomes in studies of fear of childbirth: a systematic review. Women Birth. 2019;32(2):99-111. doi:10.1016/j.wombi.2018.07.004

24. 23.Karabulutlu O, Calik KY, Akar A. Fear of childbirth and related obstetric variables. Nursing Practice Today. 2016;3(4):161-170.

25. Tofighi Niaki M, Behmanesh F, Mashmuli F, Azimi H. The Effect of Prenatal Group Education on Knowledge, Attitude and Selection of Delivery Type in Primiparous Women. Iranian Journal of Medical Education. 2010;10(2):124-130. [Persian].

(C) 2020 The Author(s); This is an open-access article distributed under the terms of the Creative Commons Attribution License (http:// creativecommons.org/licenses/by/4.0), which permits unrestricted use, distribution, and reproduction in any medium, provided the original work is properly cited. 